\title{
Does Pyloric-ring Resection in Pancreaticoduodenectomy Prevent Delayed Gastric Emptying?
}

\author{
Tortrakoon Thongkan, M.D., Thakerng Pitakteerabundit, M.D., Nan-ak Wiboonkhwan, M.D. \\ Department of Surgery, Faculty of Medicine, Prince of Songkla University, Hat Yai, Songkhla 90110, Thailand. \\ Received 15 July 2020 • Revised 21 September 2020 • Accepted 24 September 2020 • Published online 2 March 2021
}

\begin{abstract}
Objective: Delayed gastric emptying (DGE) is a common, post pancreaticoduodenectomy (PD) complication, which prolongs hospital stay, increases the cost of treatment and delays adjuvant therapy. Although, pylorospasm is one of the proposed mechanisms, the results of pyloric ring resection PD remain controversial. Hence, this study investigated whether pyloric ring resection PD decreased the incidence of DGE.

Material and Methods: Between June, 2015 and July, 2018; 74 patients underwent a PD for periampullary lesions, of whom 25 patients received pylorus-preserving PD (PPPD) and 49 patients received pyloric-ring resection PD (PRPD). DGE was classified according to the International Study Group of Pancreatic Surgery.

Results: The incidence of DGE in the PPPD group and PRPD group were 48.0\% (12 of 25 patients) and $20.4 \%$ (10 of 49 patients), respectively ( $p$-value=0.029). Factors associated with DGE were pyloric preserving [adjusted Odds ratio $(\mathrm{OR})=8.26,95 \%$ confidence interval $(\mathrm{Cl})$ : 1.96-34.82, $\mathrm{p}$-value=0.002], preoperative biliary drainage (adjusted OR=0.19, 95\% Cl: 0.05-0.78, p-value=0.013) and postoperative intraabdominal collection (adjusted OR=37, 95\% Cl: 5.68-241.24, p-value<0.001).
\end{abstract}

Conclusion: PRPD demonstrated a significant decrease of DGE, and should be one of the standard surgical treatments for periampullary carcinoma.

Keywords: delayed gastric emptying, pancreaticoduodenectomy, pyloric-ring resection

Contact: Tortrakoon Thongkan, M.D.

Department of Surgery, Faculty of Medicine, Prince of Songkla University,

Hat Yai, Songkhla 90110, Thailand.

E-mail: ktrakoon@gmail.com, ttortrak@medicine.psu.ac.th

This is an open access article under the CC BY-NC-ND license

(http://www.jhsmr.org/index.php/jhsmr/about/editorialPolicies\#openAccessPolicy).

J Health Sci Med Res 2021;39(4):303-312 doi: 10.31584 /jhsmr.2021786 www.jhsmr.org 


\section{Introduction}

Pancreaticoduodenectomy (PD) and pyloruspreserving pancreaticoduodenectomy (PPPD) are complex surgical procedures, which are indicated for periampullary carcinoma along with other, certain benign conditions; such as chronic pancreatitis and cystic neoplasm of the pancreas. Even though the mortality rate is less than $5.0 \%$, in a high volume center, it is still associated with significant morbidity. There is no significant difference between PD and PPPD in terms of hospital mortality and perioperative morbidity ${ }^{1}$, including postoperative pancreatic fistula; which is the most dreaded complication after PD. Moreover, PPPD has been reported to reduce dumping, diarrhea, and bile reflux gastritis following gastrectomy. In addition it also affords patients an improved nutritional status compared with PD., Although PPPD has been accepted as a standard surgical approach, many studies have reported on the incidence of delayed gastric emptying (DGE) to be more frequent in the PPPD group. ${ }^{4}$

DGE affects $14.0-30.0 \%$ of postoperative patients, and has been associated with increased hospital length of stay, increased costs, hospital readmission and significant patient discomfort. ${ }^{5-7}$ There are several definitions of DGE; however, the widely used International Study Group of Pancreatic Surgery (ISGPS) has classified DGE into three groups ( $A, B$ and $C$ ), with each being reflexive of the postoperative hospital stay. ${ }^{8,9}$ In the United States, median hospital charges increase by over $\$ 10,000$ with each severity grade of DGE, according to the definition of the ISGPS. ${ }^{10}$

The exact pathogenesis of DGE is still unclear; although, functional obstructions caused by stomach dysrhythmia due to vagal denervation and pylorospasm, is one of the proposed mechanisms. ${ }^{11,12}$ Hence, the concept of pyloric ring resection was introduced, so as to preserve the reservoir function of the stomach, and to simultaneously contend with the problem of pylorospasm. Pyloric-ring resection pancreaticoduodenectomy (PRPD) was first introduced in Japan in $1990^{13}$, and since then several studies were conducted to compare the postoperative outcome between PPPD and PRPD. Most of the studies reported that the overall postoperative complications and rates of pancreatic fistula formation were equivalent among the two groups. ${ }^{4}$ However, there are conflicting results in the reports of DGE outcomes between the two groups. ${ }^{13,14}$ Herein, this study was conducted to compare the rate of DGE in patients who underwent either PPPD or PRPD.

\section{Material and Methods}

Patients who underwent PD in Songklanagarind Hospital, a tertiary care hospital in southern Thailand, by a hepatobiliary and pancreatic surgery team between; June 1, 2015 and July 31, 2018, were stratified to PRPD and PPPD groups. The PPPD groups were performed between 2015 and 2016, and the PRPD groups were performed later on. Ethical approval was obtained from the Human Research Ethical Committee. Data were collected from the electronic medical records from all patients. Indication for surgery included: carcinoma of the head of the pancreas, carcinoma of distal common bile duct, carcinoma of ampulla of Vater, carcinoma of the second part of the duodenum, intraductal papillary neoplasm of the pancreas, pancreatic neuroendocrine tumors and chronic pancreatitis. Tumor invasion to the distal stomach or intraoperative diagnosis of metastatic disease were excluded. Computed tomography was performed preoperatively for all patients. Preoperative biliary drainage was performed in selected patients, who developed cholangitis or severe biliary obstructive symptoms. Preoperative details including: serum albumin, total serum bilirubin, diabetic status, preoperative biliary drainage, pancreatic duct diameter and preoperative cholangitis, were recorded. Operative time, operative blood loss and blood 
transfusions were all collected as intraoperative information. The days of hospital stay, postoperative morbidity and mortality were then compared.

The days until liquid and soft diet, as well as reinsertion of the nasogastric tube, were recorded to stratify patients into delayed gastric emptying status according to the ISGPS definition. ${ }^{8}$ A Jackson-Pratt drain was placed until the seventh day after surgery and subsequently removed, if there was no evidence of postoperative pancreatic fistula (POPF); according to the International Study Group of Pancreatic Fistula definition (ISGPF). ${ }^{15}$

Surgeries were performed by a single team of surgeons, using the same technique and approach, so as to avoid technical bias. Epidural anesthesia was introduced in all cases, if no contraindication, in order to decrease systemic opioids that might affect gastroparesis. Skin incisions were performed with an upper midline incision for optimal exposure. The duodenum transection site was 2 $\mathrm{cm}$ distal to the pylorus, for PPPD, and just proximal to the pylorus, for PRPD (Figure 1). Lymph node (LN) dissection was performed as standard lymphadenectomy for PD; as described by ISGPS ${ }^{16}$, in which LN station numbers 5,6 , $8 \mathrm{a}, 12 \mathrm{~b}, 12 \mathrm{c}, 13 \mathrm{a}, 13 \mathrm{~b}, 14,17 \mathrm{a}$ and 17b were dissected. The anastomosis reconstruction was performed using one jejunal limb, in which pancreaticojejunostomy was performed by duct-to-mucosa technique. Wherein, a 5-0 polyglactin interrupted and Silastic tube 5-8 Fr was applied across the pancreaticojejunal anastomosis as an internal stent. Two patients were reconstructed via the Dunking technique, due to the surgeons not being able to identify the pancreatic duct; hepaticojejunostomy was reconstructed by 4-0 polyglactin interrupted stitches. The gastrojejunostomy in PRPD and duodenojejunostomy in PPPD were performed as ante colic fashion, with 3-0 polyglactin at $50 \mathrm{~cm}$ distal to the hepaticojejunostomy anastomosis. One close suction drain was placed at the surgical bed.

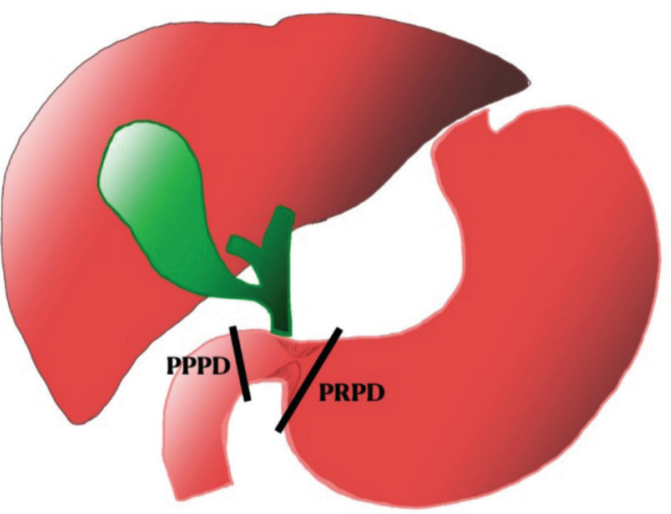

Figure 1 The duodenum transection site was $2 \mathrm{~cm}$ distal to the pylorus, for pylorus-preserving pancreaticoduodenectomy (PPPD), and just proximal to the pylorus, for pyloric-ring resection pancreatico duodenectomy (PRPD)

Patients were transferred either to the recovery room or intensive care unit, depending on their postoperative status. Metoclopramide $10 \mathrm{mg}$ intravenous injections were administered every 8 hours to all participants in the first three days, postoperatively. The protocol to remove the nasogastric tube was scheduled on the first day at any amount of content. An oral liquid diet was allowed on postoperative day (POD) 1, this was then stepped up to a soft diet on POD2, followed by a regular diet later; if the patient had good tolerance. The nasogastric tube was reinserted if the patient had clinical abdominal distension or vomiting. Serum amylase, ascites amylase, serum bilirubin and ascites bilirubin were collected on the seventh day after surgery, so as to detect pancreatic fistula and to document biliary leakage.

We calculated the sample size using two independent proportion tests, in reference to Hayashibe at el. study ${ }^{17}$, in that: 22 patients in the PPPD group and 44 patients in the PRPD group would be required in order to detect a difference in the rate of delayed gastric emptying (two- 
tailed test; alpha level, 0.05; beta level, 0.20). Additionally, an extra $10.0 \%$ of the sample size was added, in case of data loss; 25 patients in the PPPD group and 49 patients in the PRPD group. All of these were retrieved for analysis. Continuous variables were report as the mean \pm standard deviation, and compared between the two groups using an independent t-test. The discrete variables were reported as a percentage, and were compared using the Chi-squared test, $p$-values less than 0.05 were considered statistically significant. Multivariate logistic regression analysis was performed to control patient's demographic, and tumor characteristics when determining risk factors for DGE. All statistical analysis was performed by the R program, version 4.0.2.

The primary endpoint was the delayed gastric emptying, classified into three grades according to the definition proposed by the ISGPS, which impact on clinical outcome.

Grade A: Intubation of nasogastric tube required for 4-7 days, or reinsertion of the nasogastric tube after POD3 or inability to tolerate a solid diet by POD7.

Grade B: Intubation of nasogastric tube required for 8-14 days, or reinsertion of the nasogastric tube after POD7 or inability to tolerate a solid diet by POD14.

Grade C: Intubation of nasogastric tube required for 15 days, or reinsertion of the nasogastric tube after POD14 or inability to tolerate a solid diet by POD 21

The secondary endpoints were: postoperative hospital stay, intraoperative blood loss, intraoperative blood transfusion, operative time and postoperative complications; comprising of postpancreatectomy hemorrhage defined according to the definition of ISGPS. ${ }^{18}$ This included three grades (grade A, B, and C). Pancreatic fistula, defined according to the definition of ISGPF $^{15}$, including three grades (grade A, B, and C). Biliary leakage was defined according to the definition of the International Study Group of Liver Surgery (ISGLS) ${ }^{19}$, and included three grades (grade
A, B, and C). Intraabdominal collection was defined as the presence of postoperative intraabdominal fluid, confirmed by computed tomography or ultrasonography that required drainage. Wound infection was defined as surgical site infection according to the definition of the Centers for Disease Control and Prevention. Sepsis was defined as a positive bacterial culture detected from hemoculture. Pulmonary complications were defined as pneumonia or respiratory failure requiring mechanical ventilator support.

\section{Results}

From June, 2015 through till July, 2018 a total of 74 patients were enrolled; 25 patients in the PPPD group and 49 patients in the PRPD group. All patient baseline characteristics were well balanced in both study groups (Table 1). The mean age of the patients was 63 years in both groups; with most of the patients having been diagnosed with cancer. There were no differences between the two study groups, with respect to any demographic variables. Additionally, proportion of preoperative biliary drainage, preoperative cholangitis, and American Society of Anesthesiologist classification, except for the patients in the PRPD group, had significantly smaller pancreatic duct diameter ( $\leq 3$ millimeters).

The overall incidence of DGE was $29.7 \%$ (22 of 74 patients). The incidence of DGE in the PPPD group and PRPD group were $48.0 \%$ (12 of 25 patients) and $20.4 \%$ (10 of 49 patients), respectively, which had statistically significant difference $(p-$ value $=0.029)$ (Table 2). There was a significantly shorter duration of nasogastric tube insertion in the PRPD group (1 day) as compared with the PPPD group (3 days). However, duration to the start of both liquid and solid diets was comparable in both groups. Reinsertion of the nasogastric tube was $32.0 \%$ (8 of 25 patients) in the PPPD group and $16.3 \%$ (8 of 49 patients) in the PRPD group; however, the differences did not reach statistical significance. The incidence of DGE grade $A$, 
Table 1 Baseline demographic data and clinical characteristics

\begin{tabular}{|c|c|c|c|}
\hline Variable & $\begin{array}{l}\text { PPPD } \\
(n=25)\end{array}$ & $\begin{array}{l}\text { PRPD } \\
(n=49)\end{array}$ & p-value \\
\hline Age (year), mean, ( \pm S.D.) & $63.3 \pm 10.7$ & $62.5 \pm 10.3$ & 0.759 \\
\hline Male gender, n (\%) & $15(60.0)$ & $23(46.9)$ & 0.414 \\
\hline Malignant disease, $\mathrm{n}(\%)$ & $22(88.0)$ & $45(91.8)$ & 0.682 \\
\hline Primary disease, $\mathrm{n}(\%)$ & & & 0.881 \\
\hline Pancreatic adenocarcinoma & $5(20.0)$ & $10(20.4)$ & \\
\hline Bile duct carcinoma & $3(12.0)$ & $10(20.4)$ & \\
\hline Ampullary carcinoma & $12(48.0)$ & $22(44.9)$ & \\
\hline Duodenal adenocarcinoma & $0(0.0)$ & $1(2.0)$ & \\
\hline Intraductal papillary mucinous neoplasm & $2(8.0)$ & $1(2.0)$ & \\
\hline Pancreatic neuroendocrine tumor & $1(4.0)$ & $2(4.1)$ & \\
\hline Chronic pancreatitis & $0(0.0)$ & $1(2.0)$ & \\
\hline Others & $2(8.0)$ & $2(4.1)$ & \\
\hline Serum albumin, $\mathrm{g} / \mathrm{dL}$, mean, ( \pm S.D.) & $4.0 \pm 0.6$ & $3.9 \pm 0.6$ & 0.623 \\
\hline Serum total bilirubin, mg/dL, (median, IQR) & $2(0.8-4.4)$ & $1.3(0.5-6.8)$ & 0.375 \\
\hline Hemoglobin level, g/dL, mean, ( \pm S.D.) & $12.3 \pm 1.5$ & $11.8 \pm 1.8$ & 0.272 \\
\hline Preoperative biliary drainage, $\mathrm{n}(\%)$ & $13(52.0)$ & $27(55.1)$ & 0.995 \\
\hline Pancreatic duct diameter, mm, (median, IQR) & $4(3.0-6.0)$ & $3(2.0-4.0)$ & 0.054 \\
\hline Pancreatic duct diameter $\leq 3 \mathrm{~mm}, \mathrm{n}(\%)$ & $8(32.0)$ & $34(69.4)$ & 0.005 \\
\hline Preoperative cholangitis, $\mathrm{n}(\%)$ & $4(16.0)$ & $8(16.3)$ & 1.000 \\
\hline Diabetes mellitus, $\mathrm{n}(\%)$ & $6(24.0)$ & $10(20.4)$ & 0.955 \\
\hline Serum creatinine, mg\%, (median, IQR) & $0.8(0.6-0.9)$ & $0.8(0.6-0.9)$ & 0.672 \\
\hline BMI, $\mathrm{kg} / \mathrm{m}^{2}$, mean, $( \pm$ S.D. $)$ & $22.5 \pm 2.5$ & $23.0 \pm 3.8$ & 0.580 \\
\hline $\mathrm{BMI}, \mathrm{n}(\%)$ & & & 0.198 \\
\hline$<18.5 \mathrm{~kg} / \mathrm{m}^{2}$ & $1(4.0)$ & $4(8.2)$ & \\
\hline $18.5-24.99 \mathrm{~kg} / \mathrm{m}^{2}$ & $21(84.0)$ & $31(63.3)$ & \\
\hline$\geq 25 \mathrm{~kg} / \mathrm{m}^{2}$ & $3(12.0)$ & $14(28.6)$ & \\
\hline ASA classification, $\mathrm{n}(\%)$ & & & 0.458 \\
\hline ASA I & $0(0.0)$ & $2(4.1)$ & \\
\hline ASA ॥ & 17 (68.0) & $37(75.5)$ & \\
\hline ASA III & $8(32.0)$ & $10(20.4)$ & \\
\hline
\end{tabular}

S.D.=standard deviation, $I Q R=$ interquartile range, $\mathrm{g} / \mathrm{dL}=\mathrm{grams}$ per deciliter, $\mathrm{mg} / \mathrm{dL}=$ milligrams per deciliter, $\mathrm{BMl}=\mathrm{body} \mathrm{mass}$ index, $\mathrm{kg} / \mathrm{m}^{2}=$ kilogram per square meter, ASA=American Society of Anesthesiologist classification

grade $\mathrm{B}$, and grade $\mathrm{C}$ in the PPPD group was $20.0 \%$ (5 of 25 patients), $12.0 \%$ (3 of 25 patients) and $16.0 \%$ (4 of 25 patients), respectively. While, the incidence of DGE grade A, grade B and grade $\mathrm{C}$ in the PRPD group was $8.2 \%$ (4 of 49 patients), $4.1 \%$ (2 of 49 patients) and $8.2 \%$ (4 of 49 patients), respectively.

The average operative times, blood loss and incidences of perioperative blood transfusion were also comparable between the two groups. The median postoperative hospital stay was eight days in both groups. Additionally, there was no significant difference in the rates of all postoperative complications (Table 3). Death occurred in 3 of the 74 patients in the two groups combined $(4.1 \%$, two patients in the PPPD group, and one patient in the PRPD group). Only one patient had an operative-related death, which was caused by postpancreatectomy hemorrhaging; all others were related to myocardial infarction and pulmonary embolism. 
Table 2 Primary and major secondary outcomes

\begin{tabular}{|c|c|c|c|c|}
\hline Variable & $\begin{array}{l}\text { PPPD } \\
(n=25)\end{array}$ & $\begin{array}{l}\text { PRPD } \\
(n=49)\end{array}$ & $95 \% \mathrm{Cl}$ & $p$-value \\
\hline \multicolumn{5}{|l|}{ Primary outcome } \\
\hline Delayed gastric emptying, n (\%) & $12(48.0)$ & $10(20.4)$ & $28(4,45)$ & 0.029 \\
\hline DGE grade, $\mathrm{n}(\%)$ & & & & 0.088 \\
\hline A & $5(20.0)$ & $4(8.2)$ & $12(-7,26)$ & \\
\hline B & $3(12.0)$ & $2(4.1)$ & $8(-9,20)$ & \\
\hline C & $4(16.0)$ & $4(8.2)$ & $8(-11,22)$ & \\
\hline NG tube reinsertion required, n (\%) & $8(32.0)$ & $8(16.3)$ & & 0.211 \\
\hline NG tube required, days, (median, IQR) & $3(1.0-6.0)$ & $1(1.0-2.0)$ & & 0.002 \\
\hline Start of liquid diet(days), (median, IQR) & $3(3.0-6.0)$ & $3(2.0-5.0)$ & & 0.073 \\
\hline Start of solid diet(days), (median, IQR) & $4(4.0-11.0)$ & $4(3.0-6.0)$ & & 0.189 \\
\hline \multicolumn{5}{|l|}{ Secondary outcome } \\
\hline Operative time, min, mean, ( \pm S.D.) & $485.1 \pm 77.5$ & $472.8 \pm 88.2$ & & 0.556 \\
\hline Operative blood loss, mL, (median, IQR) & $400(250.0-700.0)$ & $500(300.0-800.0)$ & & 0.214 \\
\hline Blood transfusion, n (\%) & $5(21.7)$ & $15(30.6)$ & & 0.616 \\
\hline Pancreaticojejunostomy anastomotic technique, n (\%) & & & & 0.111 \\
\hline Duct to mucosa technique & $23(92.0)$ & $49(100.0)$ & & \\
\hline Dunking technique & $2(8.0)$ & $0(0.0)$ & & \\
\hline Presence of ascites, n (\%) & $0(0.0)$ & $5(10.2)$ & & 0.160 \\
\hline Postoperative hospital stay, days, (median, IQR) & $8(7.0-15.0)$ & $8(7.0-13.0)$ & & 0.792 \\
\hline
\end{tabular}

$\mathrm{DGE}=$ delayed gastric emptying, S.D.=standard deviation, $\mathrm{IQR}=$ interquartile range

Table 3 Postoperative complication

\begin{tabular}{|c|c|c|c|}
\hline Variable & $\begin{array}{l}\text { PPPD } \\
(n=25)\end{array}$ & $\begin{array}{l}\text { PRPD } \\
(n=49)\end{array}$ & $p$-value \\
\hline Postoperative pancreatic fistula, n (\%) & $5(20.0)$ & $12(24.5)$ & 0.887 \\
\hline POPF grade, $n(\%)$ & & & 1.000 \\
\hline A & $4(16.0)$ & 9 (18.3) & \\
\hline $\mathrm{B}$ & $1(4.0)$ & $3(6.1)$ & \\
\hline C & $0(0.0)$ & $0(0.0)$ & \\
\hline Bile leakage, n (\%) & $1(4.0)$ & $0(0.0)$ & 0.338 \\
\hline Postpancreatectomy hemorrhage, n (\%) & $1(4.0)$ & $1(2.0)$ & 1.000 \\
\hline PPH grade, $n(\%)$ & & & 0.565 \\
\hline A & $0(0.0)$ & $0(0.0)$ & \\
\hline B & $0(0.0)$ & $1(2.0)$ & \\
\hline $\mathrm{C}$ & $1(4.0)$ & $0(0.0)$ & \\
\hline Intraabdominal collection, n (\%) & $4(16.0)$ & $10(20.4)$ & 0.761 \\
\hline Surgical site infection, n (\%) & $5(20.0)$ & $6(12.2)$ & 0.492 \\
\hline Re-operation, n (\%) & $2(8.0)$ & $2(4.1)$ & 0.600 \\
\hline Sepsis, n (\%) & $3(12.0)$ & 8 (16.3) & 0.740 \\
\hline Pulmonary complication, n (\%) & $3(12.0)$ & $1(2.0)$ & 0.109 \\
\hline Mortality, n (\%) & $2(8.0)$ & $1(2.0)$ & 0.262 \\
\hline Operative-related & $1(4.0)$ & $0(0.0)$ & \\
\hline Non-operative-related & $1(4.0)$ & $1(2.0)$ & \\
\hline
\end{tabular}

POPF=postoperative pancreatic fistula, $\mathrm{PPH}=$ postpancreatectomy hemorrhage 
Table 4 Regression analysis

\begin{tabular}{|c|c|c|c|c|}
\hline \multirow{2}{*}{ Variable } & \multicolumn{2}{|c|}{ Univariate analysis } & \multicolumn{2}{|c|}{ Multivariate analysis* } \\
\hline & Crude OR $(95 \% \mathrm{Cl})$ & $p$-value & Adjust OR (95\% Cl) & $p$-value \\
\hline PPPD vs. PRPD & $3.60(1.26-10.27)$ & 0.015 & $8.26(1.96-34.82)$ & 0.002 \\
\hline Preoperative biliary drainage & $0.36(0.13-1.00)$ & 0.051 & $0.19(0.05-0.78)$ & 0.013 \\
\hline P-duct diameter $>3 \mathrm{~mm}$ & $0.50(0.18-1.44)$ & 0.193 & - & - \\
\hline Operative time (min) & $1.00(0.99-1.01)$ & 0.134 & - & - \\
\hline Diabetes & $0.74(0.21-2.61)$ & 0.636 & - & - \\
\hline $\mathrm{BMI} \geq 25 \mathrm{~kg} / \mathrm{m}^{2}$ & $1.05(0.14-8.02)$ & 0.403 & - & - \\
\hline POPF & $1.40(0.44-4.42)$ & 0.572 & - & - \\
\hline Intraabdominal collection & $10.00(2.67-37.47)$ & $<0.001$ & $37.00(5.68-241.24)$ & $<0.001$ \\
\hline SSI & $3.52(0.95-13.14)$ & 0.061 & - & - \\
\hline Sepsis & $5.60(1.44-21.78)$ & 0.011 & - & - \\
\hline Pulmonary complication & $8.05(0.79-82.24)$ & 0.054 & - & - \\
\hline
\end{tabular}

PPPD=pylorus-preserving pancreaticoduodenectomy, PRPD=pyloric-ring resection pancreaticoduodenectomy, BMI=body mass index, $\mathrm{POPF}=$ postoperative pancreatic fistula, $\mathrm{SSI}=$ surgical site infection

*Factors from univariate analysis, with a p-value $<0.2$, and factors, which are literately related were calculated with backward stepwise regression analysis, for optimal AIC value, then multivariate analysis was conducted.

The results of the logistic regression analysis, to identify variables associated with DGE, are provided in Table 4. Pyloric preserving [adjusted odds ratio $(\mathrm{OR})=$ 8.26, 95\% confidence interval $(\mathrm{Cl}): 1.96-34.82$, p-value= 0.002 ], preoperative biliary drainage (adjusted $\mathrm{OR}=0.19$, 95\% Cl: $0.05-0.78, p-$ value $=0.013$ ) and postoperative intraabdominal collection (adjusted OR=37, 95\% Cl: 5.68241.24, p-value $<0.001$ ) were independent factors predicting DGE by multivariate logistic analysis.

\section{Discussion}

In this present study, PRPD was significantly decreasing the DGE rate compared to PPPD (20.0\% vs. $48.0 \%$ ), respectively. The DGE rate in the PRPD groups was lower when compared to the results from the latest RCTs ${ }^{20}$ (overall DGE for PRPD group=31.2\%), which investigated the effect of pylorus resection to DGE outcomes. The duration of the NG tube requirement in PRPD groups was significantly shorter when compared to PPPD groups (1 day vs. 3 days). In our center, the NG tube was routinely removed at postoperative day 1 , not according to the volume of gastric content. However, it was re-inserted if the patient developed nausea, vomiting, or abdominal distension. Although, there were no statistically significance, the PPPD groups tended to need more NG tube re-insertion.

Many factors were proposed that relate to DGE. Most of them were postoperative complications, which directly correlated with the rate of POPF. In our study, there was no significant difference in major postoperative complications that included POPF, intraabdominal collection, and septic complications among both groups. Furthermore, the clinical pancreatic leakage (POPF grade B) were merely $4.0 \%$ and $6.1 \%$ in PPPD and PRPD groups, respectively. None of the patients was classified as POPF grade $\mathrm{C}$ in this cohort. These results supposed to exclude the effect of postoperative complications on the DGE rate in this study.

The pathogenesis of DGE is still unclear. Many factors were reported to increase the risk of DGE, for examples general preoperative condition, body mass index ${ }^{20}$, obstructive jaundice, technical aspect (preservation 
of pylorus, ante colic gastrojejunostomy) and postoperative complications (sepsis, intraabdominal collection). There are a lot of potential, physiologic changes following PD, especially the removal of the duodenum, and alterations in the normal path of the gastrointestinal flow were considered to increase DGE. The duodenectomy reduces the plasma level of motilin that predominately originates from the duodenum, resulting in delayed gastric emptying (gastroparesis), by reducing coordinated stomach, duodenum, and proximal jejunum movements. ${ }^{21}$ Our study could identify risk factors that escalated the DGE rate, including the presence of pylorus, intraabdominal collection; whereas, preoperative biliary drainage showed that it alleviates the chance of developing DGE.

Pylorospasm is one of the proposed mechanisms of DGE. The concept of pyloric ring resection was introduced to reduce the occurrence of DGE, preserving as much of the stomach as possible, so as to maintain its gastric pooling ability. Moreover, PRPD may preserve the motor innervation of the body of the stomach, which, along with the absence of pylorus, may precipitate gastric emptying. ${ }^{11}$ Many retrospective studies and $3 \mathrm{RCTs}^{13,14,20}$ were conducted to evaluate the role of pyloric ring resection $P D$ on DGE outcomes, but the results are still controversial. There are only 2 Japanese RCTs ${ }^{13,14}$ that evaluated DGE, according to the ISGPS, and these results are still in controversy as well. The limitation of these studies was the small sample size, and one of them ${ }^{14}$ used retro colic gastroenteric anastomosis, which might have affected the rate of DGE; although, the impact of the ante or retro colic reconstruction on DGE is still under debate. Furthermore, the recently published RCT from the Heidelberg group ${ }^{20}$, which is the largest in case numbers, showed no statistically significance between PPPD and PRPD. One can argue that: the overall DGE rate from the Heidelberg group was surprisingly high compared to the Japanese RCTs. From our results, the
PRPD group had a DGE rate at $20.4 \%$, which is the same as the Japanese RCTs. It also demonstrated that PRPD was statistically significant in decreasing the DGE rate compared to PPPD.

Preoperative biliary drainage was identified as the factor associated with a reduction of DGE in a meta-analysis from Qu et al. ${ }^{22}$ Our results, from multivariate analysis, also support this finding. The possible explanation for this phenomenon was that preoperative hyperbilirubinemia is considered an important risk factor for postoperative complications. Poor clearance of endotoxin from obstructive jaundice might have an effect on intestinal motility. ${ }^{23}$

The intraabdominal collection has been associated with the development of DGE postoperatively. Many retrospective studies demonstrated that postoperative pancreatic fistula and intraabdominal collection were independently associated with DGE. ${ }^{5,9}$ Intraabdominal collection, and infection caused gastroparesis and intestinal ileus. Additionally, many studies had a high pancreatic fistula rate that inevitably affects the rate of DGE. Moreover, the results from those studies cannot determine if DGE did or did not occur secondarily to these complications. Our study shows consistent results with other studies, in which intraabdominal collection is the most influential, independent predictor for the development of DGE.

There are several limitations to our study. Firstly, we relied on clinical judgment, as to whether the patient could or could not tolerate a meal. However, the most relevant functional test in a measurement of gastric emptying is gastric emptying scintigraphy. ${ }^{24}$ Secondly, some patients might develop DGE from gastrojejunostomy or duodenojejunostomy anastomosis swelling, whether from secondary intraabdominal collection or surgical technique per se. However, the simplest way to prove this by endoscopy is risky, due to the early postoperative period. 


\section{Conclusions}

Although, the development of DGE in postoperative PD patients is likely to be multi-factorial, pyloric ring resection is one of the modifiable factors that can lead to a significant decrease for the risk of DGE. In so saying, PRPD should be considered as one of the standard surgical treatments.

\section{Conflict of interest}

None declared.

\section{References}

1. Diener MK, Knaebel H-P, Heukaufer C, Antes G, Buchler MW, Seiler CM. A systematic review and meta-analysis of pylorus-preserving versus classical pancreaticoduodenectomy for surgical treatment of periampullary and pancreatic carcinoma. Ann Surg 2007;245:187-200.

2. Traverso LW, Longmire WP. Preservation of the pylorus in pancreaticoduodenectomy. Surg Gynecol Obstet 1978;146: 959-62.

3. Jimenez RE, Fernandez-del Castillo C, Rattner DW, Chang Y, Warshaw AL. Outcome of pancreaticoduodenectomy with pylorus preservation or with antrectomy in the treatment of chronic pancreatitis. Ann Surg 2000;231:293-300.

4. Fujii T, Kanda M, Kodera Y, Nagai S, Sahin TT, Hayashi M, et al. Preservation of the pyloric ring has little value in surgery for pancreatic head cancer: a comparative study comparing three surgical procedures. Ann Surg Oncol 2012; 19:176-83.

5. Parmar AD, Sheffield KM, Vargas GM, Pitt HA, Kilbane EM, Hall BL, et al. Factors associated with delayed gastric emptying after pancreaticoduodenectomy. HPB 2013;15:76372.

6. Tanaka M. Gastroparesis after a pylorus-preserving pancreatoduodenectomy. Surg Today 2005;35:345-50.

7. Ahmad SA, Edwards MJ, Sutton JM, Grewal SS, Hanseman DJ, Maithel SK, et al. Factors influencing readmission after pancreaticoduodenectomy: a multi-institutional study of 1302 patients. Ann Surg 2012;256:529-37.

8. Wente MN, Bassi C, Dervenis C, Fingerhut A, Gouma DJ,
Izbicki JR, et al. Delayed gastric emptying (DGE) after pancreatic surgery: a suggested definition by the International Study Group of Pancreatic Surgery (ISGPS). Surgery 2007;142: 761-8.

9. Malleo G, Crippa S, Butturini G, Salvia R, Partelli S, Rossini $\mathrm{R}$, et al. Delayed gastric emptying after pylorus-preserving pancreaticoduodenectomy: validation of International Study Group of Pancreatic Surgery classification and analysis of risk factors. HPB 2010;12:610-8.

10. Eisenberg JD, Rosato EL, Lavu H, Yeo CJ, Winter JM. Delayed gastric emptying after pancreaticoduodenectomy: an analysis of risk factors and cost. J Gastrointest Surg 2015;19:157280.

11. Panwar R, Pal S. The International Study Group of Pancreatic Surgery definition of delayed gastric emptying and the effects of various surgical modifications on the occurrence of delayed gastric emptying after pancreatoduodenectomy. Hepatobiliary Pancreat Dis Int 2017;16:353-63.

12. Kim DK, Hindenburg AA, Sharma SK, Suk CH, Gress FG, Staszewski $\mathrm{H}$, et al. Is pylorospasm a cause of delayed gastric emptying after pylorus-preserving pancreaticoduodenectomy? Ann Surg Oncol 2005;12:222-7.

13. Kawai M, Tani M, Hirono S, Miyazawa M, Shimizu A, Uchiyama $\mathrm{K}$, et al. Pylorus ring resection reduces delayed gastric emptying in patients undergoing pancreatoduodenectomy: a prospective, randomized, controlled trial of pylorus-resecting versus pylorus-preserving pancreatoduodenectomy. Ann Surg 2011;253:495-501.

14. Matsumoto I, Shinzeki M, Asari S, Goto T, Shirakawa S, Ajiki $\mathrm{T}$, et al. A prospective randomized comparison between pylorus- and subtotal stomach-preserving pancreatoduodenectomy on postoperative delayed gastric emptying occurrence and long-term nutritional status: SSPPD vs. PPPD. J Surg Oncol 2014;109:690-6.

15. Bassi C, Dervenis C, Butturini G, Fingerhut A, Yeo C, Izbicki J, et al. Postoperative pancreatic fistula: an international study group (ISGPF) definition. Surgery 2005;138:8-13.

16. Tol JAMG, Gouma DJ, Bassi C, Dervenis C, Montorsi M, Adham $\mathrm{M}$, et al. Definition of a standard lymphadenectomy in surgery for pancreatic ductal adenocarcinoma: a consensus statement by the International Study Group on Pancreatic Surgery (ISGPS). Surgery 2014;156:591-600. 
17. Hayashibe A, Kameyama M, Shinbo M, Makimoto S. The surgical procedure and clinical results of subtotal stomach preserving pancreaticoduodenectomy (SSPPD) in comparison with pylorus preserving pancreaticoduodenectomy (PPPD). J Surg Oncol 2007;95:106-9.

18. Wente MN, Veit JA, Bassi C, Dervenis C, Fingerhut A, Gouma DJ, et al. Postpancreatectomy hemorrhage (PPH)-An International Study Group of Pancreatic Surgery (ISGPS) definition. Surgery 2007;142:20-5.

19. Besselink MG, van Rijssen LB, Bassi C, Dervenis C, Montorsi $M$, Adham $M$, et al. Definition and classification of chyle leak after pancreatic operation: a consensus statement by the International Study Group on Pancreatic Surgery. Surgery 2017;161: 365-72.

20. Hackert T, Probst P, Knebel P, Doerr-Harim C, Bruckner T, Klaiber
$\mathrm{U}$, et al. Pylorus resection does not reduce delayed gastric emptying after partial pancreatoduodenectomy: a blinded randomized controlled trial (PROPP Study, DRKS00004191). Ann Surg 2018;267:1021-7.

21. Kang CM, Lee JH. Pathophysiology after pancreaticoduodenectomy. World J Gastroenterol 2015;21:5794-804.

22. Qu H, Sun GR, Zhou SQ, He QS. Clinical risk factors of delayed gastric emptying in patients after pancreaticoduodenectomy: a systematic review and meta-analysis. EJSO 2013;39:213-23.

23. Nieuwenhuijs VB, van Dijk JE, Gooszen HG, Akkermans LMA. Obstructive jaundice, bacterial translocation and interdigestive small-bowel motility in rats. Digestion 2000;62:255-61.

24. Camilleri M, Chedid V, Ford AC, Haruma K, Horowitz M, Jones KL, et al. Gastroparesis. Nat Rev Dis Primer 2018;4:41-59. 